\title{
ARTROPODOFAUNA CADAVÉRICA ASOCIADA A Sus scrofa L. EN EL SUR DE URUGUAY
}

\author{
Mónica Remedios-De León, Manuel Castro \& Enrique Morelli
}

\author{
Sección Entomología, Facultad de Ciencias, Universidad de la República, Iguá 4225, C.P. 11400, Montevideo, \\ Uruguay. \\ * Autor para correspondencia: mremedios@fcien.edu.uy
}

\section{RESUMEN}

La Entomología Forense es de gran valor en casos donde los restos humanos son colonizados por insectos. Su contribución más destacada consiste en establecer el intervalo post-mortem ya que algunas de las especies de la comunidad sarcosaprófaga están asociadas a etapas concretas del proceso de descomposición. Los restos orgánicos en descomposición, tanto animales como humanos, experimentan una serie de cambios físicos, químicos y biológicos, que los van transformando en un recurso efímero nutricionalmente rico, para diferentes taxa de animales. Durante el proceso de descomposición, los órdenes Diptera, Coleoptera e Hymenoptera, son predominantes en diversidad y abundancia de individuos. El objetivo de este trabajo es presentar el primer estudio de artropodofauna cadavérica sobre modelos experimentales porcinos en un ambiente de bosque para el Sur de Uruguay. Para ello se emplearon como modelo biológico dos ejemplares de cerdo blanco (Sus scrofa). Para la captura de insectos adultos se utilizó una trampa Malaise, red entomológica y captura manual. Se recolectaron un total de 2926 individuos adultos distribuidas en tres Órdenes y 14 familias. La mayor riqueza de especies se observó en la estación de verano y en primavera la menor.

Palabras clave: Sucesión, Fauna sarcosaprofaga, Forense.

\section{ABSTRACT}

Cadaveric arthropodofauna associated to Sus scrofa L. in the south of Uruguay. Forensic Entomology is the branch of science that uses arthropods as a tool in research when classical forensic methods are insufficient. It is thus that this branch represents an invaluable aid in cases where the human remains are colonized by insects. The most important contribution of forensic entomology is associated with the estimation of the post-mortem interval since some species of the sarcosaphage community are associated with specific stages of the decomposition process. The cadaveric arthropodofauna decomposing organic remains, both animal and human, undergo a series of physical, chemical and biological changes, transforming it into a nutritionally rich but provisional resource, for different taxa of animals. During the decomposition process, the orders Diptera, Coleoptera and Hymenoptera, are predominant in diversity and abundance. The objective of this work is to present the first study of cadaveric arthropodofauna on experimental porcine models in a forest environment for Southern Uruguay. For this, two specimens of white pig (Sus scrofa L.) were used as biological model. A malaise trap, entomological net and manual capture were used for the capture of adult insects. A total of 2926 adults were collected from three orders and 14 families. The greatest species richness was observed in the summer season and in the lower spring.

Key words: Succession, Wildlife sarcosaprofaga, Forensic.

\section{INTRODUCCIÓN}

La Entomología Forense o Entomología Médicolegal, es la rama de la ciencia que emplea los artrópodos como herramienta en la investigación cuando los métodos forenses clásicos son insuficientes (Camacho, 2005). Es así como la Entomología Forense representa una ayuda invaluable en casos donde los restos humanos son colonizados por insectos. El aporte más destacado de la Entomología Forense está asociado a la estimación del intervalo post-mortem, ya que algunas de las especies de la comunidad sarcosaprófaga están asociadas a etapas concretas del proceso de descomposición. Los restos orgánicos en descomposición, tanto animales como humanos, experimentan una serie de cambios físicos, químicos y biológicos, que lo van transformando en un recurso nutricionalmente rico pero provisorio, para diferentes taxa de animales (Anderson \& VanLaerhoven, 1996; Centeno et al., 2002; Battán Horenstein et al., 2005, 2010). Esta serie de cambios que pueden variar dependiendo de las condiciones medio ambiental y el tamaño de los cuerpos, se manifiestan de manera casi continua. Anderson y VanLaerhoven (1996) mediante un modelo experimental realizado en cerdos pudieron reconocer las siguientes fases: Fresca, Enfisematosa, Descomposición Activa, Descomposición Avanzada y Restos Secos. Diferentes grupos de insectos necrófagos pueden colonizar cada 
una de estas fases. Durante el proceso de descomposición, los restos pasan por una serie de cambios biológicos, químicos y físicos, desde su estado fresco hasta la esqueletización que, en conjunto, determinan la diversidad de insectos sarcosaprófagos, así como la secuencia con la cual van colonizando los restos (Anderson \& VanLaerhoven, 1996). Una de las clasificaciones más utilizada es la de Leclercq (1978) y Goff et al. (1993) clasifican a los insectos que acuden a un cadáver en base a su alimentación dividiéndolos en especies necrófagas principalmente dípteros y coleópteros, especies necrófilas incluyendo himenópteros y algunos coleópteros que se alimentan de las especies necrófagas, especies omnívoras agrupando aquellos insectos que se alimentan de tejidos muertos y finalmente las especies oportunistas como colémbolos, crustáceos, y arañas. Durante el proceso de descomposición, los órdenes Diptera, Coleoptera e Hymenoptera, son predominantes en diversidad y abundancia (Payne, 1965; Dadour et al., 2001; Aballay et al., 2008; Byrd \& Castner, 2010). Los Diptera son los principales representante de los insectos en el proceso de descomposición y la familia Calliphoridae es la más abundante (Kitching et al., 2004; de Carvalho \& Mello-Patiu, 2008; Amendt et al., 2010; Battán et al., 2010; Vasconselos \& Araujo, 2012). Sus diferentes especies son las primeras en llegar y las más comunes en la descomposición de cadáveres en su etapa larval. Los coleópteros son el segundo orden en importancia en estudios de Entomología Forense, están presentes en todas las etapas del proceso de descomposición de un cadáver alimentándose de los fluidos y exudados de la materia orgánica en Algunas de las Familias más importantes son Dermestidae, Histeridae, Staphylinidae entre otras. La acción de estos acelera el proceso reduciendo el tiempo de permanencia del cuerpo en el suelo (Payne, 1965; Putman, 1983). En el caso de Hymenoptera los ejemplares capturados en los cuerpos se consideran necrófilos ya que pueden actuar como predadores y muchas especies son parasitoides (Castillos, 2002). Una de las familias más representativa en la Entomología Forense es Formicidae, con una presencia constante en todos los estados de descomposición. La acción de las hormigas se limita a sorber los jugos desprendidos de los cadáveres y en algún caso al transporte hacia sus hormigueros de larvas de dípteros (Castillos, 2002). En Uruguay, los estudios sobre sucesión de entomofauna cadavérica han sido muy escasos y dispersos, no existiendo datos publicados de los mismos. Este trabajo tiene como objetivo presentar el primer estudio de artropodofauna cadavérica sobre modelos experimentales porcinos en un ambiente de bosque en el Sur de Uruguay para las cuatro estaciones del año, contribuyendo con la creación de una línea de base de utilidad para el desarrollo de la Entomología Forense para el país.

\section{MATERIALES Y MÉTODOS}

El estudio se llevó a cabo en una área semirural de la ciudad de Pando en el departamento de Canelones, Uruguay (34042'37.45"S y 55058'24.27"O). Se seleccionó un predio con presencia de Eucalyptus sp. de más de cinco años. Para cubrir las cuatro estaciones del año se emplearon como modelo biológico dos ejemplares de cerdo blanco (Sus scrofa L.), según la metodología propuesta por Rodríguez \& Bass (1983); Catts \& Goff (1992); Anderson \& VanLaerhoven (1996). Los cuerpos se colocaron en posición lateral sobre mallas de metal y dentro de jaulas de madera de $1 \mathrm{~m} \mathrm{x}$ $1 \mathrm{~m} \times 1 \mathrm{~m}$, con un revestimiento de tejido con abertura de malla de $3 \mathrm{~cm}$ para protegerlos de animales carroñeros. Cada cerdo se colocó a 100 metros de distancia entre ellos y en las mismas condiciones ambientales (sobre suelo de tierra y expuestos al sol la mayor parte del día). Los muestreos se realizaron durante los años 2015 y 2016. Las muestras se tomaron cada 24 horas durante la primera semana, luego cada 48 horas, posteriormente cada 72 horas y semanalmente a medida que la abundancia de insectos iba disminuyendo. Para la captura de insectos adultos se utilizó una trampa Malaise (Townes, 1962), colocada dentro de la jaula cubriendo el cuerpo (Castillos, 2002; Pinto et al., 2010; Aballay et al., 2011). También se realizó captura con red entomológica y recolección manual directa sobre, debajo y alrededor del cadáver. Los insectos se sacrificaron con acetato de etilo y se conservaron en seco en sobres de papel. Para la determinación taxonómica hasta el nivel de especie se emplearon las claves de Mariluis \& Peris (1984); Smith (1986); Oliva (2002); Amat et al. (2008); Nihei \& Domínguez (2008); Aballay et al. (2013); Whitworth (2014). Los datos meteorológicos se tomaron de la página del Instituto Nacional Uruguayo de Meteorología (InUMet) y las temperaturas corporales internas de los cerdos con un termómetro digital de pincho. Se realizaron análisis de Kruskal-Wallis para observar diferencias significativas y dendrogramas de similitud para comparar la entomofauna en las distintas estaciones del año. También se realizó un análisis SIMPER (Clarke \& Gorley, 2001) de correspondencia para determinar la asociación de las especies de insectos a cada estación e identificar las especies tipificantes y las discriminantes para cada estación. Se utilizó el Índice de Shannon para explorar la variación en la diversidad de especies. Un lote de referencia de cada especie se depositó en la Colección de Entomología de la Facultad de Ciencias de la Universidad de la República.

\section{RESULTADOS}

La temperatura media para la estación de verano fue de $21.8^{\circ} \mathrm{C} \pm 0.41$, para otoño $10.7^{\circ} \mathrm{C} \pm 0.34$, 
Tabla 1. Temperaturas medias ambientales y corporales de los cerdos en cuatro periodos estacionales. (X sin datos).

\begin{tabular}{|c|c|c|c|c|c|c|c|c|}
\hline & $\begin{array}{l}\text { PRIMAVERA } \\
\text { T. amb. }{ }^{\circ} \mathrm{C}\end{array}$ & $\begin{array}{l}\text { CERDOS } \\
\text { T. Interna }{ }^{\circ} \mathrm{C}\end{array}$ & $\begin{array}{l}\text { VERANO } \\
\text { T. amb. }{ }^{\circ} \mathrm{C}\end{array}$ & $\begin{array}{l}\text { CERDOS } \\
\text { T. Interna }{ }^{\circ} \mathrm{C}\end{array}$ & $\begin{array}{l}\text { INVIERNO } \\
\text { T. amb. }{ }^{\circ} \mathrm{C}\end{array}$ & $\begin{array}{l}\text { CERDOS } \\
\text { T. Interna }{ }^{\circ} \mathrm{C}\end{array}$ & $\begin{array}{c}\text { OTOÑO } \\
\text { T. amb. }{ }^{\circ C}\end{array}$ & $\begin{array}{l}\text { CERDOS } \\
\text { T. Interna }{ }^{\circ} \mathrm{C}\end{array}$ \\
\hline $\mathrm{F}$ & 14,9 & 37 & 26,3 & 37,5 & 6,6 & 38 & 17,5 & 37 \\
\hline $\mathrm{H}$ & 12,7 & 23 & 23,6 & 39 & 9,1 & 11 & 13,6 & 22 \\
\hline DAC & 13,9 & 14 & 21,6 & 22 & 10,9 & 11 & 11,6 & 12 \\
\hline $\mathrm{DAZ}$ & 15,6 & $x$ & 21,6 & $x$ & 12,4 & $x$ & 9,8 & $x$ \\
\hline $\mathrm{R}$ & 18,5 & $x$ & 20,5 & $x$ & 12,5 & $X$ & 9,1 & $X$ \\
\hline
\end{tabular}

Tabla 2. Duración en días de cada una de las etapas del proceso de descomposición en cuatro periodos estacionales (F: fresco; H: hinchado; DAC: descomposición activa; DAZ: descomposición avanzada y R: restos secos).

\begin{tabular}{lccccc} 
Estación & $\mathrm{F}$ & $\mathrm{H}$ & $\mathrm{DAC}$ & $\mathrm{DAZ}$ & $\mathrm{R}$ \\
\hline Primavera & 1 & 5 & 8 & 15 & 19 \\
Verano & 1 & 3 & 10 & 16 & 5 \\
Invierno & 2 & 12 & 14 & 39 & 9 \\
Otoño & 2 & 10 & 16 & 27 & 17 \\
\hline
\end{tabular}

invierno $11.5^{\circ} \mathrm{C} \pm 0.33$ y para primavera $16.2^{\circ} \mathrm{C} \pm$ 0.42. En la Tabla 1 se presenta las temperaturas ambientales con relación a las temperaturas registradas en el interior de los cuerpos, para las cuatro estaciones del año. Las temperaturas internas al comienzo del experimento fueron mayores a la ambiental en las cuatro estaciones explicada esto por la propia temperatura de los cuerpos, disminuyendo paulatinamente hasta alcanzar los niveles de las temperaturas ambientales. Las precipitaciones medias registradas para cada estación fueron de $82 \mathrm{~mm}$ para verano, $94 \mathrm{~mm}$ para otoño, $73 \mathrm{~mm}$ para invierno y 78 $\mathrm{mm}$ para primavera. En la descomposición activa la temperatura media interna de los cuerpos alcanzó los niveles de temperatura ambiental. Estados de descomposición: Se observaron las cinco etapas del proceso de descomposición descritas por Anderson y VanLaerhoven (1996): Fresco (F), Hinchado $(H)$, Descomposición activa (DAC), Descomposición avanzada (DAZ) y Restos (R). La duración total del proceso de descomposición fue mayor durante el invierno (76 días), seguido por otoño (72), primavera (48 días), y finalmente con una duración de 35 días para la estación de verano. La etapa fresca fue la de menor duración en días para las cuatro estaciones del año. La duración de la fase hinchada fue de 12 días para invierno, 10 para otoño, cinco para primavera y de tres días para verano. La fase de descomposición activa registró el menor valor en días (8) en primavera y el máximo (16 días) en otoño. La fase de descomposición avanzada tuvo una mayor duración en invierno (39 días) y para la fase de restos podemos visualizar una duración de 5 y 9 días para las estaciones de verano y de 17 y 19 para otoño y primavera (Tabla 2). Se recolectaron un total de 2926 individuos adultos distribuidos en tres Ordenes y 14 familias: Diptera (Calliphoridae, Sarcophagidae, Muscidae, Fanniidae, Piophilidae y Anthomyiidae) (66\%), Hymenoptera (Formicidae) (24\%) y Coleoptera (Staphylinidae, Histeridae, Cleridae, Dermestidae, Trogidae, Silphidae) (10\%) (Tabla 3). La mayor riqueza de especies se observó en la estación de verano (36) y en primavera la menor (22). Los valores más altos del índice de Shannon se obtuvieron durante el invierno (2697) los valores más bajos en verano (1249). Mediante el Test de Kruskal-Wallis y Bray-Curtis observamos que la composición de especies no fue similar en las cuatro estaciones del año $(P=0.0171)$ (Tabla 4) (Fig. 1). EI SIMPER identificó las especies tipificantes de cada estación del año: Chrysomya albiceps Wiedemann, 1819, está asociada a la estación de verano, Calliphora vicina Robineau-Desvoidy, 1830, al invierno, Euspilotus azureus Sahlberg, 1823, a la primavera, Fanniidae sp. 1 fue la única especie tipificante para el otoño. (Tabla 5).

\section{DISCUSIÓN Y CONCLUSIÓN}

Las etapas del proceso de descomposición halladas en el presente trabajo son similares a las de otros estudios para la región (Wolff et al., 2001; Martínez et al., 2006; Segura et al., 2011; Armani et al., 2015). Calliphora vicina fue la especie tipificante para invierno. Es una especie ampliamente distribuida y de clima frío por lo que las experiencias en países de climas cálidos no la registran en su elenco de especies (Smith, 
Tabla 3. Abundancias absolutas ( $\mathrm{n}^{\circ}$ de individuos) de los taxa de insectos capturados (I: invierno; P: primavera: V: verano y O: otoño)

\begin{tabular}{|c|c|c|c|c|c|c|}
\hline \multirow{2}{*}{ Ordenes } & \multirow{2}{*}{ Familias } & \multirow[t]{2}{*}{ Especies } & \multicolumn{4}{|c|}{ Estaciones } \\
\hline & & & $\mathbf{I}$ & $\mathbf{P}$ & V & 0 \\
\hline \multirow[t]{39}{*}{ Diptera } & Calliphoridae & Chrysomya albiceps Wiedemann, 1819 & 0 & 4 & 1120 & 23 \\
\hline & & Chrysomya chloropyga Wiedemann, 1818 & 0 & 0 & 3 & 1 \\
\hline & & Chrysomya megacephala Fabricius, 1794 & 0 & 0 & 5 & 0 \\
\hline & & Lucilia peruviana Robineau-Desvoidy, 1830 & 1 & 2 & 0 & 0 \\
\hline & & Lucilia cuprina Wiedemann, 1830 & 2 & 0 & 0 & 0 \\
\hline & & Lucilia sericata Meigen, 1826 & 1 & 13 & 0 & 76 \\
\hline & & Sarconesia chlorogasterWiedemann, 1830 & 2 & 1 & 0 & 9 \\
\hline & & Cochliomyia macellaria Fabricius, 1775 & 0 & 0 & 3 & 0 \\
\hline & & Calliphora vicina Robineau-Desvoidy, 1830 & 47 & 1 & 0 & 7 \\
\hline & Sarcophagidae & Oxysarcodexia varia Walker, 1836 & 0 & 0 & 29 & 1 \\
\hline & & Oxysarcodexia terminalis Wiedemann, 1830 & 0 & 0 & 9 & 0 \\
\hline & & Oxysarcodexia culmiforceps Dodge, 1966 & 0 & 0 & 9 & 0 \\
\hline & & Oxysarcodexia paulistensis Mattos, 1919 & 0 & 0 & 12 & 1 \\
\hline & & Ravinia advena Walker, 1853 & 0 & 0 & 4 & 0 \\
\hline & & Ravinia sueta Wulp, 1895 & 0 & 0 & 10 & 0 \\
\hline & Muscidae & Ophyra albuquerquei Lopes, 1985 & 0 & 1 & 0 & 0 \\
\hline & & Ophyra chalcogaster Wiedemann, 1824 & 3 & 3 & 3 & 3 \\
\hline & & Morellia violacea Robineau-Desvoidy, 1830 & 1 & 0 & 0 & 0 \\
\hline & & Psilochaeta chalybea Wiedemann, 1830 & 0 & 0 & 0 & 2 \\
\hline & & Psilochaeta pampiana Shannon \& Del Ponte, 1926 & 2 & 0 & 0 & 0 \\
\hline & & Gymnodia cuadristigma Thomson, 1869 & 1 & 0 & 0 & 0 \\
\hline & & Hydrotaea sp. Robineau-Desvoidy, 1830 & 1 & 4 & 0 & 1 \\
\hline & & Neomuscina zosteri Shannon \& Del Ponte, 1926 & 1 & 0 & 0 & 0 \\
\hline & & Neurotrixia felsina Walker, 1849 & 9 & 5 & 0 & 0 \\
\hline & & Muscina stabulana Fallén, 1817 & 5 & 3 & 2 & 8 \\
\hline & & Musca domestica Linnaeus, 1758 & 0 & 0 & 0 & 7 \\
\hline & Fanniidae & Fanniidae sp & 0 & 0 & 0 & 67 \\
\hline & & Fannia canicularis Linnaeus, 1761 & 1 & 0 & 16 & 1 \\
\hline & & Fannia fusconatata Rondani ,1868 & 1 & 0 & 5 & 1 \\
\hline & & Fannia heydenni Stein, 1911 & 0 & 0 & 1 & 1 \\
\hline & & Fannia sanihue Dominguez \&Aballay, 2008 & 0 & 3 & 259 & 25 \\
\hline & Phoridae & Megaselia rufipes Meigen, 1804 & 0 & 0 & 1 & 0 \\
\hline & & Megaselia scalaris Loew, 1866 & 3 & 0 & 1 & 0 \\
\hline & Anthomyiidae & Anthomyiidae punctipennis Wiedemann ,1830 & 7 & 1 & 0 & 0 \\
\hline & Piophilidae & Piophila casei Linnaeus, 1758 & 0 & 0 & 15 & 0 \\
\hline & & Atheta sp.Thomson, 1858 & 3 & 6 & 0 & 0 \\
\hline & & Lathrobium sp. Gravenhorst,1802 & 0 & 0 & 3 & 0 \\
\hline & & Platydracus sp.Thomson, 1858 & 0 & 0 & 5 & 0 \\
\hline & & Rugilus sp.Samouelle, 1819 & 0 & 0 & 5 & 0 \\
\hline \multirow[t]{9}{*}{ Coleoptera } & Histeridae & Carcinops (C) troglodytes Paykull, 1811 & 0 & 0 & 3 & 0 \\
\hline & & Euspilotus (H) azureus Sahlberg, 1823 & 10 & 45 & 14 & 16 \\
\hline & & Euspilotus (H) connectens Paykull, 1811 & 0 & 4 & 4 & 3 \\
\hline & & Euspilotus (H) modestus Erichson ,1834 & 2 & 0 & 4 & 1 \\
\hline & & Euspilotus sp. 1 Lewis, 1807 & 0 & 0 & 2 & 0 \\
\hline & & Phelister rufinotus Marseul, 1861 & 0 & 0 & 4 & 0 \\
\hline & Cleridae & Necrobia rufipes DeGeer, 1775 & 4 & 0 & 1 & 12 \\
\hline & & Necrobia ruficollis Fabricius, 1775 & 0 & 2 & 0 & 0 \\
\hline & Dermestidae & Dermestes maculatus DeGeer, 1774 & 2 & 8 & 10 & 5 \\
\hline
\end{tabular}


Tabla 3. (Continuación)

\begin{tabular}{|c|c|c|c|c|c|c|}
\hline \multirow[t]{5}{*}{ Ordenes } & \multirow[t]{2}{*}{ Familias } & \multirow[t]{2}{*}{ Especies } & \multicolumn{4}{|c|}{ Estaciones } \\
\hline & & & $\mathbf{I}$ & $\mathbf{P}$ & V & $\mathbf{0}$ \\
\hline & Silphidae & Oxelytrum discicolle Brullé, 1836 & 6 & 0 & 0 & 0 \\
\hline & Trogidae & Trogidae Morfoespecie 1 & 2 & 0 & 0 & 0 \\
\hline & & Polynoncus sp. Burmeister, 1847 & 1 & 0 & 0 & 0 \\
\hline \multirow[t]{8}{*}{ Hymenoptera } & Formicidae & Acromyrmex sp. Mayr, 1865 & 25 & 15 & 95 & 10 \\
\hline & & Ectatomma sp. 1 Smith, 1858 & 1 & 3 & 5 & 2 \\
\hline & & Pachycondyta striata Smith, 1858 & 0 & 0 & 1 & 0 \\
\hline & & Pheidole sp. 1 Westwood, 1839 & 71 & 92 & 270 & 52 \\
\hline & & Pheidole sp. 2 Westwood, 1839 & 12 & 15 & 28 & 12 \\
\hline & & Pogonomyrmex sp. 1 Mayr, 1868 & 1 & 0 & 1 & 0 \\
\hline & & Pseudomirmex sp. 2 Lund, 1831 & 1 & 0 & 1 & 1 \\
\hline & & Solenopsis sp. 1 Westwood, 1840 & 1 & 0 & 1 & 0 \\
\hline
\end{tabular}

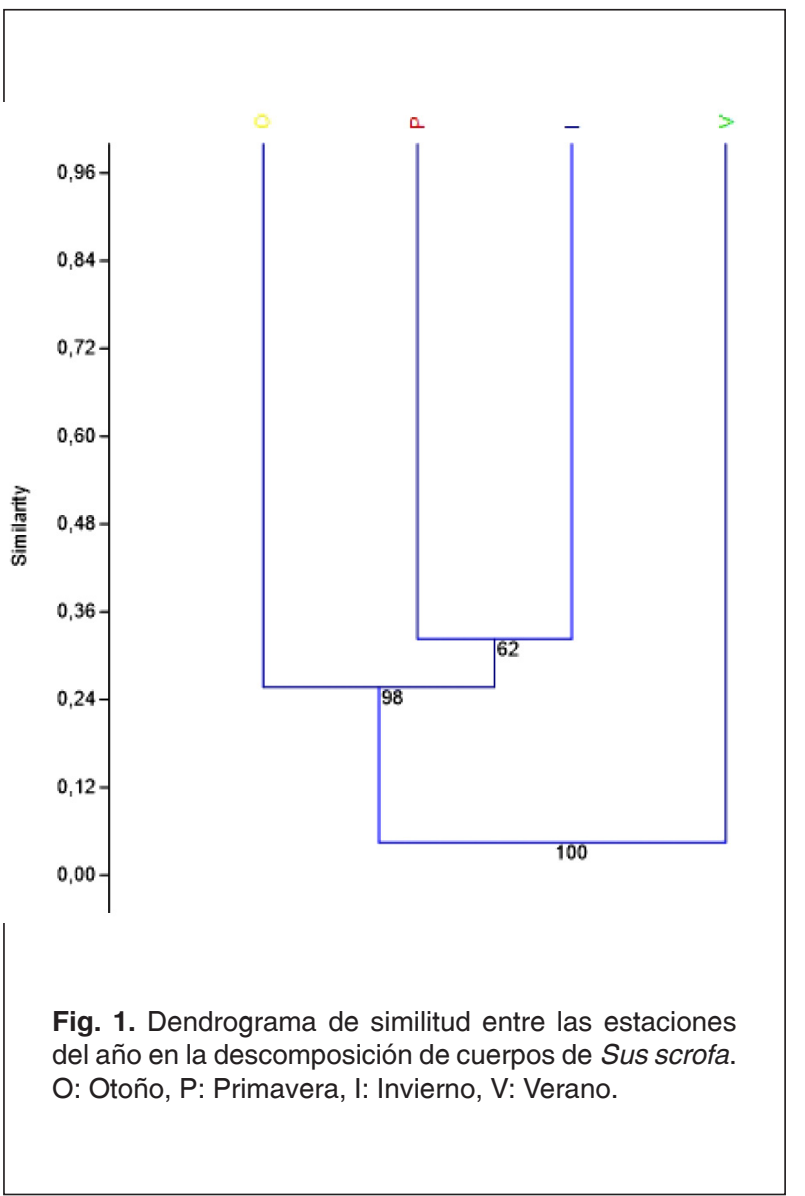

Tabla 4. Test de Kruskal-Wallis para las cuatro estaciones de año.

\begin{tabular}{lcccc} 
& Invierno & Primavera & Verano & Otoño \\
\hline Invierno & 0 & 0,1527 & 0,05245 & 0,5106 \\
Primavera & 0,9163 & 0 & 0,002933 & 0,4523 \\
Verano & 0,3147 & 0,0176 & 0 & 0,0366 \\
\hline Otoño & $\mathbf{1}$ & $\mathbf{1}$ & $\mathbf{0 , 2 1 9 6}$ & $\mathbf{0}$ \\
\hline
\end{tabular}

Tabla 5. Porcentajes de contribución de las especies a los promedios de similitud dentro de cada estación del año (especies tipificantes $>10 \%$ ).

Especies Tipificantes Invierno Primavera Verano Otoño
C. vicina
33,62
E. azureus
45,55
Ch. albiceps
74,81

Fanniidae sp.1

Similitud promedio $\quad 67,41 \quad 84,41 \quad 83,48 \quad 73,33$ 
1986). Este califórido es considerado como colonizador primario dominante en las regiones subtropicales en las estaciones frías llegando al cadáver durante los primeros minutos después de la muerte y si las condiciones ambientales son adecuadas, pueden registrarse las primeras posturas (Tantawi et al., 1996; lannacone, 2000; Wolff et al., 2001; Arnaldos et al., 2004; Moneo Pellitero \& Saloña Bordas, 2009). Lucilia sericata Meigen, 1826 registra la mayor abundancia en la estación de otoño coincidiendo con los resultados de Armani et al. (2015) para una localidad del sur de Argentina, sin embargo esta especie fue citada como especie de verano para experiencias similares en el Norte de Argentina (Aballay et al., 2008). Chrysomya albiceps de acuerdo con los análisis SIMPER fue la especie tipificante para el verano. Coincidiendo con los registros de esta especie para la mayoría de los países de América del Sur asociando esta especie con las altas temperaturas (Ortloff-Trautmann et al., 2013). Investigaciones similares en países de clima frío no reportaron la presencia de esta especie (Saiz et al., 1989; Figueroa-Roa \& Linhares, 2002; OrtloffTrautmann et al., 2013). Autores como Faria y cols. (1999) han observado que su introducción al Nuevo Mundo ha tenido un fuerte impacto en la abundancia de dípteros endémicos. Es probable que ello esté favorecido por la capacidad depredadora facultativa y el comportamiento agresivo de esta especie durante su etapa larvaria, llegando a alterar la composición de especies de dípteros dentro del cadáver (Castillos, 2002). Antes de la introducción de Ch. albiceps, Cochliomyia macellaria Fabricius, 1775 era considerada una especie dominante en áreas semirurales de América (Schnack et al., 1995). La baja proporción encontrada de $C$. macellaria en este estudio con sólo tres ejemplares adultos registrados, que concuerda con las bajas abundancias observadas para la provincia de Buenos Aires (Centeno et al., 2002). Los registros de Sarconecia chlorogaster Wiedemann, 1830 son coincidentes con otros trabajos para climas fríos de Sudamérica y en particular su ausencia para la estación de verano (Aballay et al., 2008). Sarcophagidae fue la Familia de interés forense con abundancia más baja. Esto puede deberse a la competencia natural entre las larvas de Calliphoridae y Sarcophagidae (Catts \& Goff 1992; Castillos, 2002). La baja abundancia y estacionalidad de las especies Oxisarcodexia varia Walker, 1836 y Oxisarcodexia paulistensis Mattos, 1919 también son reportadas para otros estudios en Argentina (Armani et al., 2015). Ophyra chalcogaster Lopes, 1985 presente durante todo el año, difiere con los resultados obtenidos para el Sur de Argentina (Armani et al., 2015). Muscina stabulans Fallén, 1817 y Musca domestica Linnaeus, 1758 se caracterizan por sus bajos registros. En cuanto a la estacionalidad, para nuestra experiencia $M$. domestica solo fue registrada en otoño diferenciándose de otros trabajos para la región con presencia durante las cuatro estaciones (Wolff et al., 2001; Armani et al., 2015). Domínguez \& Gómez (1963) la citan como una especie ocasional que se alimenta de los exudados y sangre de los cuerpos. Fannia sanihue Dominguez \& Aballay, 2008 y Fanniidae sp. fueron las dos especies más abundantes. Fannia sanihue fue descrita por primera vez en Mendoza, Argentina (Domínguez \& Aballay, 2008) y en 2014 para Uruguay. En cuanto a las abundancias y estacionalidad de los coleópteros Dermestes maculatus DeGeer, 1774 y Euspilotus modestus Erichson, 1834 no se apartan de los resultados obtenidos para otros estudios de sucesión en cerdo blanco para América del Sur. D. maculatus es citado tanto para ambientes urbanos como rurales o semirurales (Vasconcelos \& Araujo, 2012) y ha sido reportado por varios autores como buen indicador de las últimas etapas en los procesos de descomposición (Smith, 1986; Oliveira-Costa et al., 2001; Carvalho et al., 2004; Almeida \& Mise, 2009; Byrd \& Castner, 2010). E. azureus tuvo una permanencia constante durante las cuatro estaciones, estos resultados muy similares a los obtenidos por García-Rojo (2004); por otra parte, Vasconcelos y Araujo (2012) citan al género Euspilotus como exclusivo de ambientes rurales. Entre lo himenópteros, los formícidos Acromyrmex sp. y Pheidole sp. fueron los géneros con mayores registros durante todo el año con mayor abundancia en el verano. Las hormigas desprenden pequeños trozos de tejido y piel de los cadáveres además de sorber los jugos desprendidos (Byrd \& Castner, 2010) pudiéndose también alimentar de huevos y larvas de dípteros o transportarlos hacia sus hormigueros (Castillos, 2002). Pheidole ha sido reportado por Centeno (2002) como importante género necrófilo. Si bien las especies de Acromyrmex son de hábitos cortadores, las obreras no se alimentan exclusivamente del hongo que cultivan, sino que también obtienen nutrientes de jugos vegetales, es probable que también se alimenten de los fluidos del cadáver (Byrd \& Castner, 2010).

\section{BIBLIOGRAFÍA}

Aballay F., Arriagada G., Flores G. \& N. Centeno. 2013. An illustrated key to and diagnoses of the species of Histeriade (Coleoptera) associated with decaying carcasses in Argentina. ZooKey, 261: 61-84.

Aballay F., Fernández Campón F., Mulieri P. \& S. Urquiza. 2011. Sarcophagidae (Diptera) de importancia forense en la puna de Catamarca, Argentina: ovoviviparidad como ventaja en condiciones de extrema aridez. Revista Sociedad Entomológica Argentina, 70(3-4): 255266.

Aballay F., Murúa A., Acosta J.C. \& N. Centeno. 2008. Primer registro de artropodofauna cadavérica en sustratos humanos y animales en San Juan, 
Argentina. Revista Sociedad Entomológica Argentina, 67(3-4): 157-163.

Almeida L.M. \& K.M. Mise. 2009. Diagnosis and key of the main families and species of South American Coleoptera of forensic importance. Revista Brasileña de Entomologia, 53: 227-244.

Amat E., Velez M. \& M. Wolff. 2008. Clave ilustrada para la identificación de los géneros y las especies de califóridos (Diptera: Calliphoridae) de Colombia. Caldasia, 30: 231-244.

Amendt J.C., Campobasso L. \& M. Grassberger. 2010. Current concepts in Forensic Entomology. London: Springer Dordrecht Heidelberg, 121pp.

Anderson G. \& S.L. Vanlaerhoven. 1996. Initial Studies on Insect Succession on Carrion in Southwestern British Columbia. Journal of Forensic Sciences, 41: 617-625.

Armani A.P., Centeno N. \& L. Silva. 2015. Primer estudio de artropodofauna cadavérica sobre modelos experimentales porcinos en el noreste de la provincia del Chubut, Argentina. Revista de la Sociedad Entomológica Argentina, 74(34): 123-132.

Arnaldos M.I., Romera E., Presa J.J., Luna A. \& M.D. García. 2004. Studies on seasonal arthropod succession on carrion in the southeastern iberian Peninsula. International Journal of Legal Medecine, 118(4): 197-205.

Battán Horenstein M., Arnaldos M., Rosso B. \& M. García. 2005. Estudio preliminar de la comunidad sarcosaprófaga en Córdoba (Argentina): aplicación a la entomología forense. Anales de Biología, 27: 191-201.

Battán Horenstein M., Linhares A.X., Rosso De Ferradas B. \& M. García. 2010. Decomposition and dipteran succession on pig carrion in central Argentina: ecological aspects and their importance to forensic science. Medical and Veterinary Entomology, 24: 16-25.

Byrd J.H. \& J.L. Castner. 2010. Forensic Entomology: the utility of arthropods in legal investigations, 2nd ed, Boca Raton, CRC Press, 705 pp.

Camacho G. 2005. Sucesión de la entomofauna cadavérica y ciclo vital de Calliphora vicina (Diptera: Calliphoridae) como primera especie colonizadora, utilizando cerdo blanco (Sus scrofa) en Bogotá, 31: 189-197.

Carvalho L.M.L., Thyssen P.J., Goff M.L. \& A.X. Linhares. 2004. Observations on the succession patterns of necrophagous insects onto a pig carcass in a urban area of Southeastern Brazil. Anal Aggrawal's Internet Journal of Forensic Medicine and Toxicology, 5: 33-39.

Castillos M. 2002. Estudio de la entomofauna asociada a cadáveres en el Alto Aragón (España). Monografías Sociedad Entomológica Aragonesa. $96 \mathrm{pp}$.

Catts E.P. \& M.L. Goff. 1992. Forensic entomology in criminal investigations. Annual Reviews of Entomology, 37: 253-272.

Centeno N. 2002. Experimentos de campo sobre sucesión de fauna cadavérica. Actas y Trabajos del V Congreso Argentino de Entomología. Argentina. 67-69 pp.

Centeno N., Maldonado M. \& A. Oliva. 2002. Seasonal patterns of arthropods occurring on sheltered and unsheltered pig carcasses in Buenos Aires Province (Argentina). Forensic Science International, 126: 63-70.

Clarke K.R. \& R.N. Gorley. 2001. PRIMER. Version 5: User manual tutorial. Plymouth: Plymouth Marine Laboratory.

Dadour I.R., Cook D.F., Fissioli J.N. \& W.J. Bailey. 2001. Forensic entomology: application, education and research in Western Australia. Forensic Science International, 120(1-2): 4852.

de Carvalho C.J.B. \& C.A. Mello-Patiu. 2008. Key to the adults of the most common forensic species of Diptera in South America. Revista Brasileira de Entomologia, 52: 390-406.

Dominguez M.C. \& F. Aballay. 2008. A new species of the genus Fannia Robineau-Desvoidy (Diptera: Fanniidae) collected on pig carrion in Mendoza, Argentina. Annals of Zoology, 58: 819-824.

Domínguez J. \& L. Gómez. 1963. Momificación cadavérica particularmente rápida, operada bajo la acción de numerosas larvas de Chrysomya albiceps, Wiedemam, 1819. Revista Ibérica de Parasitología, Tomo XXIII, no 1-2: 43-62.

Faria L.D.B., Orsi L., Trinca L.A. \& W. Godoy. 1999. Larval predation by Chrysomya albiceps on Cochliomyia macellaria, Chrysomya megacephala, and Chrysomya putoria. Entomologia Experimentalis Applicata, 90: 149155.

Figueroa-Roa L. \& A.X. Linhares. 2002. Sinantropia de los Calliphoridae (Diptera) de Valdivia, Chile. Neotropical Entomology, 31: 233-239.

García-Rojo A. 2004. Estudio de la sucesión de insectos en cadáveres en Alcalá de Henares (Comunidad Autónoma de Madrid) utilizando cerdos domésticos como modelos animales. Boletín de la Sociedad Entomológica Aragonesa, 34: 263-269.

Goff M.L., Brown W.A., Omori A.I. \& D.A. Lapointe. 1993. Prelimanry observations of the effects of Amytriptyline in decomposing tissues on the development of Parasarcophaga ruficornis (Diptera: Sarcophagidae) and implications of this effect to estimation of postmortem interval. Journal of Forensic Science, March 1993: 316322.

Iannacone J. 2000. Artropofauna de importancia forense asociada a un cadáver de cerdo en Ventanilla - Callao, Perú. Revista Brasileira de Zoología, 20: 85- 90. 
InUMet. Instituto Uruguayo de Meteorología. En línea:http://www.meteorologia.com.uy.

Kitching R.L., Bickel D., Creagh A.C., Hurley K. \& C. Symonds. 2004. The biodiversity of Diptera in Old World rain forest surveys: a comparative faunistic analysis. Journal of Biogeography, 31 : 1185-1200.

Leclercq, M. 1978. Entomologie et Médecine légale. Datation de la Mort. Collection de Médecine légale et de Toxicologie médicale, $\mathrm{n} \square 108$. Masson, Paris, 100 pp.

Mariluis J.C. \& S. Peris. 1984. Datos para la sinopsis de los Calliphoridae Neotropicales. Revista Española de Entomología, 50: 67-86.

Martínez E., Duque P. \& M. Wolff. 2006. Succession pattern of carrion-feeding insects in Paramo, Colombia. Forensic Science International, 166: 182-189.

Moneo Pellitero J. \& M.I. Saloña Bordas 2009. Primera cita de Chrysomya megacephala (Fabricius) (Díptera: Calliphoridae) en la Comunidad Autónoma del País Vasco. Boletín de la Sociedad Entomológica Aragonesa, 44: 528-530.

Nihei S. \& M.C. Domínguez. 2008. Muscidae. En: Claps L.E., Debandi G. \& S. Roig-Juñent (Eds.) Biodiversidad de Artrópodos Argentinos, pp. 319-328. Vol. 2. Mendoza, Argentina.

Ortloff-Trautmann A., Jara-Peñailillo A., AlbornozMuñoz S., Silva-Riveros R., Riquelme-Gatica M. \& P. Peña-Rehbein. 2013. Primer reporte en Chile de Chrysomya albiceps (Diptera: Calliphoridae) en evidencia entomológica forense. Archivos de Medicina Veterinaria, 45: 83-89.

Oliva A. 2002. Diptera (Insecta) de interés forense o causantes de Miasis. Claves artificiales para estadios preimaginales. En: Salomón O.D. (Ed.) Actualizaciones en antropología sanitaria Argentina, pp. 51-59. Fundación Mundo Sano.

Oliveira-Costa J., Mello-Patiu C.A. \& S.M. Lopes. 2001. Muscoid diptera associated with human corpses at the death scene in the State of Rio de Janeiro, Brazil. Boletin do Museu Nacional, N.S. (Zool) 464, 1-6.

Payne J.A. 1965. A summer carrion study of the baby pig (Sus scrofa). Ecology, 46: 592-602.

Pinto J, Ferreira R., Carvalho C. \& P. Ribeiro. 2010. Evidence of the influence of Malaise trap age on its efficiency in the collection of Muscidae
(Insecta, Diptera). International Journal of Tropical Insect Science, 30 (2): 115-118.

Putman R. 1983. Carrion and Dungs. The descomposition of animal Wastes.Edward Arnold, London, $61 \mathrm{pp}$.

Rodríguez W.C. \& W.M. Bass. 1983. Insect activity and its relation to decay rates of human cadavers in east Tennessee. Journal Forensic Science, 28: 423-432.

Saiz F., Tosti-Croce E. \& M. Leiva. 1989. Estudio de los cambios de la mesofauna asociada a la descomposición de cadáveres de conejo en clima mediterráneo. Annales of Museum History Natural Valparaiso, 20: 41-74.

Schnack J.A, Mariluis J.C, Centeno N. \& J. Muzón. 1995. Composición específica, ecología y sinantropía de Calliphoridae (Insecta: Diptera) en el Gran Buenos Aires. Revista de la Sociedad Entomológica Argentina, 54 (1-4): 161-171.

Segura N., Bonilla M., Usaquén W. \& F. Bello. 2011. Entomofauna resource distribution associated with pig cadavers in Bogotá DC. Medical and Veterinary Entomology, 25: 46-52.

Smith, K. 1986. A Manual of Forensic Entomology. The Trustees of the British Museum, 205 pp.

Tantawi T.I., EL-Kady E.M., Greenberg B. \& H.A. ELGhaffar. 1996. Arthropod succession on exposed rabbit carrion in Alexandria, Egypt. Journal of Medical Entomology, 33(4): 566-580.

Townes, H. 1962. Design of a Malaise Trap. Procedures of the Entomological Society of Washington.Tropical rainforest on Oahu Island, Hawaii. Journal Medical Entomology, 24: 332339.

Vasconcelos S. \& M. Araujo. 2012. Necrophagus species of Diptera y Coleoptera in northeastern the forensic entomologist. Revista Brasileira de Entomologia, 56(1): 7-14.

Whitworth T. 2014. A revision of the Neotropical species of Lucilia Robineau-Desvoidy (Diptera: Calliphoridae). Zootaxa, 3810 (1): 1-76.

Wolff M., Uribe A., Ortiz A. \& P. Duque. 2001. A preliminary study of forensic entomology in Medellín, Colombia. Forensic Science International, 120: 53-59.

Fecha de Recepción: 27 de febrero de 2019 Fecha de Aceptación: 11 de junio de 2019 